\title{
Probing Non-Toric Geometry with Rotating Membranes
}

\author{
Jung Hun Lee, Sunchang Kim, Jongwook Kim, and Nakwoo Kim \\ Department of Physics and Research Institute of Basic Science, \\ Kyung Hee University, Seoul 130-701, Korea
}

(Dated: November 4, 2018)

\begin{abstract}
Recently Martelli and Sparks presented the first non-toric $\mathrm{AdS}_{4} / \mathrm{CFT}_{3}$ duality relation between M-theory on $\mathrm{AdS}_{4} \times V_{5,2} / \mathbb{Z}_{k}$ and a class of three-dimensional $\mathcal{N}=2$ quiver Chern-Simons-matter theories. $V_{5,2}$ is a seven-dimensional homogeneneous Sasaki-Einstein manifold with isometry group $S O(5) \times U(1)_{R}$, which is in general broken to $S U(2) \times U(1) \times U(1)_{R}$ by the orbifold projection if $k>1$. The dual field theory is described by the $\mathcal{A}_{1}$ quiver, $U(N)_{k} \times U(N)_{-k}$ gauge group, four bifundamentals, two adjoint chiral multiplets interacting via a cubic superpotential. We explore this proposal by studying various classical membrane solutions moving in $V_{5,2}$. Rotating membrane solutions of folded, wrapped, spike, and giant magnon types are presented with their dispersion relations. We also discuss their dual operators in the Chern-Simons-matter theory.
\end{abstract}

PACS numbers: 11.25.Yb, 11.25.Tq

Keywords: M-theory, membrane, Chern-Simons theory

* nkim@khu.ac.kr 


\section{INTRODUCTION}

Over the last two years there has been remarkable progress on the understanding of M2brane dynamics. It is now widely accepted that multiple M2-branes can be described by Chern-Simons-matter theories. More concretely, the most well-established relation dictates M2-branes at orbifold singularity $\mathbb{C}^{4} / \mathbb{Z}_{k}$ are described by $\mathcal{N}=6$ supersymmetric quiver Chern-Simons-matter theory with $U(N)_{k} \times U(N)_{-k}$ gauge symmetry and four bifundamental chiral multiplets [1]. The order of orbifold group $k$ appears as the quantized Chern-Simons level in the field theory. In terms of $\mathrm{AdS} / \mathrm{CFT}$, the dual is M-theory in $\mathrm{AdS}_{4} \times S^{7} / \mathbb{Z}_{k}$ background.

It is certainly an interesting problem to find new $\mathrm{AdS}_{4} / \mathrm{CFT}_{3}$ duals with less supersymmetry. We now have a large number of such proposals, see for instance [2-10]. One may utilize the brane construction technique [11] and write Chern-Simons duals for various orbifolds of $\mathbb{C}^{4}$. Or one turns to the brane tiling method [12, 13] if the ambient geometry is toric.

In general we expect a $\mathcal{N}=2$ superconformal field theory for a Freund-Rubin type background of $\mathrm{AdS}_{4} \times \mathcal{M}_{7}$, if $\mathcal{M}_{7}$ is a seven dimensional Sasaki-Einstein manifold. In canonical form a Sasaki-Einstein manifold is written as a twisted $U(1)$ fibration over a Kähler-Einstein manifold. $\mathbb{C P}^{3}$ leads to the trivial example of $S^{7}$, while $\mathbb{C P}^{1} \times \mathbb{C P}^{1} \times \mathbb{C P}^{1}$ and $\mathbb{C P}^{2} \times \mathbb{C P}^{1}$ lead to so-called $Q^{1,1,1}$ and $M^{1,1,1}$ respectively. Since the rank of isometry group is four, above are all examples of toric manifolds. The dual field theories are all given as quiver Chern-Simons models.

In order to establish the duality, one first computes the vacuum moduli space of the field theory. In general one recovers a discrete quotient, or an orbifold of the desired SasakiEinstein space. Then we have to check if the spectrum of chiral primary operators is consistent with Kaluza-Klein spectrum of the 11 dimensional supergravity and orbifolds thereof. It turns out to be crucial to include monopole operators in order to see the symmetry enhancement for $k=1$. For more details on $Q^{1,1,1}$ and $M^{1,1,1}$, see [3] and [8].

One can go beyond the particle limit and consider membrane dynamics directly. Although it is not known how to quantize membranes in a nontrivial background, one can use classical solutions with large energy as an approximation of the full quantum result. Such a semiclassical approach of AdS/CFT is initiated in [14], and for the applications in the context 
of M2-branes see for instance [15, 16]. One can study explicit rotating membrane solutions, which are dual to gauge invariant composite operators with large conformal dimension. The dispersion relations between energy and angular momenta are believed to provide nontrivial quantitative prediction on the conformal field theory.

We continue our previous work [16] and study rotating membranes in $\operatorname{AdS}_{4} \times V_{5,2}$ in this paper. $V_{5,2}$ is another example of seven dimensional Sasaki-Einstein manifold. It is a coset $S O(5) \times S O(2) / S O(3) \times S O(2)$ so homogeneous, but non-toric since its isometry is $U(1) \times S O(5)$ and rank 3 . The dual field theory is given as a relatively simple quiver ChernSimons theory with cubic superpotential [10]. The spectra of chiral primary operators are consistent with the Kaluza-Klein analysis of 11 dimensional supergravity on $V_{5,2}$ reported in [17].

We probe this non-toric duality using membranes. Explicit classical solutions are constructed and we provide the dispersion relations. As we wrap the membranes along the great circle of an $S^{2}$ within $V_{5,2}$, the membrane dynamics is effectively reduced to that of Polyakov strings. We study configurations analogous to folded, wrapped strings as well as giant magnons [18] and single spikes [19]. We will discuss what dual operators would look like.

This paper is organized as follows. Sec II will be a review of [10] and we present both the supergravity background and quiver Chern-Simons theories, mainly to setup the notations. Sec.III is the main part where we report on the membrane solutions and discuss the field theory interpretations. We will conclude in Sec [V] with discussions.

\section{II. $\mathbf{A d S}_{4} \times V_{5,2}$ AND THE DUAL CHERN-SIMONS THEORY}

\section{A. The supergravity background}

Let us start by presenting the M-theory background of our interest. We will closely follow [10], although occasionally we employ different conventions for later conveniece. For the geometry of $V_{5,2}$, see also [20].

The 11 dimensional metric is given as a direct product of $\mathrm{AdS}_{4}$ and a seven-dimensional 
space $V_{5,2}$.

$$
\begin{aligned}
\mathrm{d} s_{11}^{2} & =L^{2}\left(\frac{1}{4} \mathrm{~d} s_{\mathrm{AdS}_{4}}^{2}+\mathrm{d} s_{V_{5,2}}^{2}\right) \\
\mathrm{d} s_{\mathrm{AdS}_{4}}^{2} & =-\cosh ^{2} \rho \mathrm{d} t^{2}+\mathrm{d} \rho^{2}+\sinh ^{2} \rho\left(\mathrm{d} \vartheta^{2}+\sin ^{2} \theta \mathrm{d} \varphi^{2}\right) \\
\mathrm{d} s_{V_{5,2}}^{2} & =\frac{9}{64}\left[\mathrm{~d} \psi+\cos \alpha\left(\mathrm{d} \beta+\cos \theta_{1} \mathrm{~d} \phi_{1}+\cos \theta_{2} \mathrm{~d} \phi_{2}\right)\right]^{2}+\mathrm{d} s_{G r_{5,2}}^{2}
\end{aligned}
$$

In the above the Sasaki-Einstein space $V_{5,2}$ is expressed in canonical form, i.e. $U(1)$-fibration over Kähler-Einstein space. $G_{5,2}=S O(5) / S O(3) \times S O(2)$ is a coset space, so it is homogeneous and the metric can be given as

$$
\begin{aligned}
\mathrm{d} s_{G r_{5,2}}^{2}= & \frac{3}{32}\left[4 \mathrm{~d} \alpha^{2}+\sin ^{2} \alpha\left(\mathrm{d} \beta+\cos \theta_{1} \mathrm{~d} \phi_{1}+\cos \theta_{2} \mathrm{~d} \phi_{2}\right)^{2}\right. \\
& +\left(1+\cos ^{2} \alpha\right)\left(\mathrm{d} \theta_{1}^{2}+\sin ^{2} \theta_{1} \mathrm{~d} \phi_{1}^{2}+\mathrm{d} \theta_{2}^{2}+\sin ^{2} \theta_{2} \mathrm{~d} \phi_{2}^{2}\right) \\
& +2 \sin ^{2} \alpha \cos \beta \sin \theta_{1} \sin \theta_{2} d \phi_{1} \mathrm{~d} \phi_{2}-2 \sin ^{2} \alpha \cos \beta \mathrm{d} \theta_{1} \mathrm{~d} \theta_{2} \\
& \left.-2 \sin ^{2} \alpha \sin \beta\left(\sin \theta_{2} \mathrm{~d} \phi_{2} \mathrm{~d} \theta_{1}+\sin \theta_{1} \mathrm{~d} \phi_{1} \mathrm{~d} \theta_{2}\right)\right]
\end{aligned}
$$

The ranges of the coordinates are

$$
0 \leq \theta_{1}, \theta_{2} \leq \pi, \quad 0 \leq \phi_{1}, \phi_{2}<2 \pi, \quad 0 \leq \psi<4 \pi, \quad 0 \leq \alpha \leq \frac{\pi}{2}, \quad 0 \leq \beta<4 \pi
$$

One can compute the volume of $V_{5,2}$ and obtain

$$
\operatorname{Vol}\left(V_{5,2}\right)=\frac{27}{128} \pi^{4}
$$

It will be particularly important for us that and the isometry group of $V_{5,2}$ is $S O(5) \times$ $U(1)_{R}$. Since the rank of the group is three while the complex dimension of the cone over $V_{5,2}$ is four, we have a non-toric manifold.

The curvature of the above metric is sourced by a four-form flux,

$$
G^{(4)}=\frac{3 L^{3}}{8} \operatorname{Vol}\left(\mathrm{AdS}_{4}\right)
$$

The quantization of the G-flux allows us to relate $L$ with the number of M2-branes, i.e.

$$
L^{6}=\frac{\left(4 \pi l_{p}\right)^{6} N}{81 \pi^{4}}
$$

where $l_{p}$ is the eleven-dimensional Planck length. 


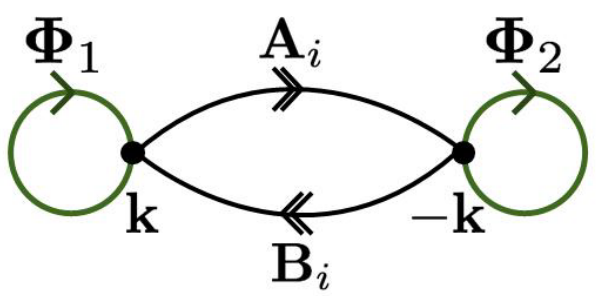

FIG. 1. The quiver diagram dual to $\mathrm{AdS} 4 \times V_{5,2}$.

\section{B. The quiver Chern-Simons-matter theory}

Now we move to the dual conformal field theory side. According to [10], the ChernSimons-matter theory described by $\mathcal{A}_{1}$ quiver in FIG. 1 is dual to M-theory in $\operatorname{AdS}_{4} \times V_{5,2} / \mathbb{Z}_{k}$. The gauge symmetry of the conformal field theory is $U(N) \times U(N)$, with Chern-Simons levels $(k,-k)$. As it is obvious in the quiver diagram, $\Phi_{1}$ and $\Phi_{2}$ are in adjoint representation of each $U(N)$. The matter fields $A_{1}, A_{2}$ are in $(N, \bar{N})$, while $B_{1}, B_{2}$ are in $(\bar{N}, N)$ representations. In total, there are thus $6 N^{2}$ chiral multiplets of $\mathcal{N}=2$ supersymmetry in three dimensions.

In addition to the gauge couplings, the interactions are described by the following cubic superpotential.

$$
W=\operatorname{Tr}\left[s\left(\Phi_{1}^{3}+\Phi_{2}^{3}\right)+\Phi_{1}\left(A_{1} B_{2}-A_{2} B_{1}\right)+\Phi_{2}\left(B_{2} A_{1}-B_{1} A_{2}\right)\right]
$$

where $s$ is a complex-valued coupling constant. Obviously all the elementary fields should be given R-charges $2 / 3$. One can easily check that $W$ is invariant under $S U(2)$ if $\left(A_{1}, A_{2}\right)$ and $\left(B_{1}, B_{2}\right)$ are both doublets. One discovers there is another $U(1)$, with charge assignments $0,1 / 2,-1 / 2$ for $\Phi_{i}, A_{i}, B_{i}$ respectively. As we will see later, for $k=1$ the non- $\mathrm{R}$ symmetry $S U(2) \times U(1)$ is enhanced to $S O(5)$.

For abelian case $N=1$, one can easily see that the F-term conditions imply

$$
\Phi_{1}+\Phi_{2}=0, \quad 3 s \Phi_{1}^{2}+A_{1} B_{2}-A_{2} B_{1}=0
$$

If one defines the new variables as $z_{1}=\frac{1}{2}\left(A_{1}+B_{2}\right), z_{2}=\frac{i}{2}\left(A_{1}-B_{2}\right), z_{3}=\frac{i}{2}\left(A_{2}+B_{1}\right), z_{4}=$ $\frac{1}{2}\left(A_{2}-B_{1}\right)$ and $z_{0}=(3 s)^{1 / 2} \Phi_{1}$, we have an equation

$$
X_{2} \equiv\left\{z_{0}^{2}+z_{1}^{2}+z_{2}^{2}+z_{3}^{2}+z_{4}^{2}=0\right\}
$$

for the vacuum moduli space. 
Obviously $X_{2}$ is a complex four dimensional space with $A_{1}$-type singularity at the origin. Like a conifold which is the three dimensional version [21], $X_{2}$ is Calabi-Yau and one can assign a Kähler and Ricci-flat metric. $X_{2}$ can be also viewed as a metric cone, and it turns out that $V_{5,2}$ is the base manifold. It is thus conjectured [10] that the quiver Chern-Simons theory in FIG. 1 has an IR fixed point which is dual to the near horizon limit of M2-branes of M2-branes at the hypersurface singularity of $X_{2}$.

In principle one has to identify the points along the gauge orbits, but the baryonic $U(1)$ gauge transformation is used to fix the phase of the dual photon field. Of course this is the same as what happens in the ABJM model [1]. For generic values of $k>1$, the gauge invariance requires we identify

$$
\left(A_{1}, A_{2}, B_{1}, B_{2}\right) \sim\left(e^{\frac{2 \pi i}{k}} A_{1}, e^{\frac{2 \pi i}{k}} A_{2}, e^{-\frac{2 \pi i}{k}} B_{1}, e^{-\frac{2 \pi i}{k}} B_{2}\right)
$$

The flavor symmetry $S U(2)_{A} \times S U(2)_{B}$ for doublets $A_{i}$ and $B_{i}$, although not manifest in the field theory, is translated to the two two-spheres parametrized by $\theta_{i}, \phi_{i}$ in $V_{5,2}$. The quotient in Eq. (12) should correspond to

$$
\left(\phi_{1}, \phi_{2}\right) \sim\left(\phi_{1}+\frac{2 \pi}{k}, \phi_{2}-\frac{2 \pi}{k}\right)
$$

It is then obvious that the isometry $S O(5)$ is broken to $S U(2) \times U(1) \subset S U(2) \times S U(2) \subset$ $S O(4) \subset S O(5)$, for $k>1$.

We are now ready to consider chiral primary operators and see how they are arranged in terms of $U(1)_{R} \times S U(2) \times U(1)$. Let us first consider abelian $N=1$ case with large $k$. As usual, we enumerate gauge singlet operators up to F-term conditions. At order one, we have only one neutral operator, $\Phi_{1}\left(\right.$ or $\left.-\Phi_{2}\right)$. This operator carries both conformal dimension and R-charge $2 / 3$, but it is a singlet and neutral for $S U(2) \times U(1)$. At order two, after taking Eq. 10 into account, we have four independent operators: $A_{1} B_{1}, A_{2} B_{2}, \frac{1}{\sqrt{2}}\left(A_{1} B_{2}+A_{2} B_{1}\right)$, and $\Phi_{1}^{2}$. The first three obviously constitute a triplet of $S U(2)$ and has $U(1)$ charge 1 . $\Phi_{1}^{2}$ is invariant under $S U(2) \times U(1)$. All of them have both conformal dimension and R-charge 4/3. One can easily convince oneself that this pattern will persist with higher order operators. At level $n$, the operators have conformal dimension and R-charge $2 n / 3$. The $S U(2)$ spin and $U(1)$ charge should take values between 0 and $n / 2$.

It is the monopole operators which play a crucial role in the symmetry enhancement for small values of $k$. The mechanism is again very similar to the ABJM model [1] and 
other examples of duality for three dimensional Chern-Simons field theories. See for instance [3, 8, 16] for homogeneous Sasaki-Einstein manifolds $Q^{1,1,1}$ and $M^{1,1,1}$. A monopole operator for abelian case is the dual photon field $e^{i a}$ which carries charges $(k,-k)$ for gauge fields $U(1) \times U(1)$. For $k=1$, we can essentially make every combination $A^{m_{1}} B^{m_{2}}$ neutral, if we allow insertions of an arbitrary number of monopole operators. Put differently, we can use the neutral combinations $A_{i} e^{-i a}, B_{i} e^{i a}$ as the new alphabets in constructing words of chiral primary operators. Under the assumption that the monopole operators do not change the conformal dimension, one obtains $S O(5)$ since there is now only the F-term condition Eq. (11) to consider.

In general for nonabelian theories with $k=1$, one can express the chiral primary operators very schematically as

$$
\operatorname{Tr}\left[\Phi^{n_{1}}(A B)^{n_{2}}\left(A e^{-i a}\right)^{m_{1}}\left(B e^{i a}\right)^{m_{2}}\right]
$$

For the comparison with rotating membranes, it is useful to record the possible $S U(2)_{A} \times$ $S U(2)_{B}$ representations in addition to the R-charge

$$
R=\frac{2}{3}\left[n_{1}+2 n_{2}+m_{1}+m_{2}\right]
$$

Since the number of $A$ 's is $n_{2}+m_{1}$, the spin for $S U(2)_{A}$ ranges up to $\left(n_{2}+m_{1}\right) / 2$. For $S U(2)_{B}$, the maximum spin is obviously $\left(n_{2}+m_{2}\right) / 2$.

\section{ROTATING MEMBRANES IN $V_{5,2}$}

As in our previous work on $\mathrm{AdS}_{4} \times M^{1,1,1}[16$, for the membrane dynamics we will use the partly gauged-fixed version of Polyakov-type action which is devised by Bozhilov in [15].

$$
S=\int \frac{\mathrm{d}^{3} \sigma}{4 \lambda^{0}}\left[G_{00}-\left(2 \lambda^{0} T_{2}\right)^{2} \operatorname{det} G_{i j}\right],
$$

where $G_{m n}=\partial_{m} X^{M} \partial_{n} X^{N} g_{M N}(X)$ is the induced metric on the membrane worldvolume. $T_{2}$ is the tension and $\lambda^{0}$ is a Lagrange multiplier. In addition to the Euler-Lagrange equations derived from Eq.(16), we have constraint equations

$$
\begin{aligned}
G_{00}+\left(2 \lambda^{0} T_{2}\right)^{2} \operatorname{det} G_{i j} & =0 \\
G_{0 i} & =0 .
\end{aligned}
$$

With some wisely chosen ansätze, the equations of motion and constraints are either trivially satisfied or derivable from an auxiliary mechanical system. In this section we study various 
nontrivial solutions of folded/wrapped rotating membranes, and also giant magnons and spinning spikes.

Since we are only interested in the membrane motion in $\mathbb{R}_{t} \times V_{5,2}$, we will set the coordinates in $\mathrm{AdS}_{4}$ to constants, i.e. $\rho=\vartheta=\varphi=0$. Furthermore, we choose the temporal gauge and set $t=\kappa \tau$. We will consider membranes spinning along $\psi, \beta, \phi_{1}, \phi_{2}$ angles. Once we find explicit solutions, we compute the angular momenta conjugate to $\psi, \phi_{1}, \phi_{2}$ and identify them as the R-charge, spins for $S U(2)_{A}$ and $S U(2)_{B}$ respectively.

\section{A. Particle-like solutions and chiral primaries}

In this section we consider membranes collapsed to a point. In other words, we will simply study the geodesic motion and evaluate the conserved quantities. It turns out that without losing generality we can set $\psi=\omega \tau$ and all other angles to arbitrary constants. One can easily check that all the equations of motion are satisfied as well as the constraints, provided $\kappa^{2}=\frac{9}{16} \omega^{2}$.

The conserved charges are given as

$$
Q_{t}=\frac{\sqrt{\lambda^{\prime}}}{4} \kappa, \quad Q_{\psi}=\frac{9 \sqrt{\lambda^{\prime}}}{64} \omega, \quad Q_{\phi_{1}}=\frac{9 \sqrt{\lambda^{\prime}}}{64} \omega \cos \alpha \cos \theta_{1}, \quad Q_{\phi_{2}}=\frac{9 \sqrt{\lambda^{\prime}}}{64} \omega \cos \alpha \cos \theta_{2},
$$

with $\sqrt{\lambda^{\prime}}=L^{2} / 2 \lambda^{0}$.

It is natural to identify $E \equiv Q_{t}$ as the conformal dimension $\Delta$ of the dual field theory operator. Then one can see that

$$
E=\frac{4}{3}\left|Q_{\psi}\right| \geq \frac{4}{3}\left|Q_{\phi_{i}}\right|
$$

which can be compared to the field theory side relation for chiral primary operators,

$$
\Delta=|R| \geq \frac{8}{3}\left|J_{A}\right|, \frac{8}{3}\left|J_{B}\right|
$$

We thus choose to identify $R=\frac{4}{3} Q_{\psi}, J_{A}=\frac{1}{2} Q_{\phi_{1}}, J_{B}=\frac{1}{2} Q_{\phi_{2}}$ from now on.

It is easy to write down the dual operators for the particle solutions with different constant values of $\alpha, \theta_{1}, \theta_{2}$. If $\kappa=3 \omega / 4>0$, the dual operators are holomorphic expressions of $\Phi, A_{i}, B_{i}$ and $E=R$. For $\alpha=\pi / 2, J_{A}=J_{B}=0$ and the dual operators should be $\operatorname{Tr} \Phi^{3 R / 2}$.

Another extreme example is at $\alpha=\theta_{1}=\theta_{2}=0$, which leads to $\Delta=R=\frac{8}{3} J_{A}=\frac{8}{3} J_{B}$. They are dual to $\operatorname{Tr}\left(A_{1} B_{1}\right)^{3 R / 4}$. 


\section{B. Simple classes of rotating wrapped membranes}

From this point on, we will consider membrane solutions occupying a nontrivial two dimensional space at every instance. Before we move to nontrivial folded/wrapped type solutions in the next subsection, we present here relatively simple solutions with a quadratic dispersion relation.

Consider the following configuration.

$$
\psi=\omega \tau, \quad \alpha=n \sigma_{1}, \quad \theta_{1}=\theta_{2}=m \sigma_{2}, \quad \beta=\pi \text { or } 3 \pi,
$$

and $\phi_{1}, \phi_{2}$ are set to arbitrary constants. The winding numbers $n, m$ are integers. All the equations are either trivially satisfied or equivalent to

$$
\kappa^{2}=\frac{9}{16} \omega^{2}+\left(2 \lambda^{0} T_{2} L\right)^{2} \frac{9 n^{2} m^{2}}{16} .
$$

It is easy to compute the conserved quantities,

$$
E=\frac{\sqrt{\lambda^{\prime}}}{4} \kappa, \quad R=\frac{3 \sqrt{\lambda^{\prime}}}{16} \omega, \quad J_{A}=J_{B}=0 .
$$

Combining these two equations, we have the dispersion relation

$$
E^{2}=R^{2}+\left(\frac{3 n m \sqrt{\lambda}}{16}\right)^{2}
$$

Note that the gauge parameter $\lambda^{0}$ disappers in the dispersion relation. We have defined $\sqrt{\lambda}=T_{2} L^{3}$, which corresponds to 't Hooft coupling constant on the dual field theory side.

One can also check that configurations given as

$$
\psi=\omega \tau, \quad \theta_{1}=\theta_{2}=m \sigma_{2}, \quad \beta=0 \text { or } 2 \pi,
$$

with

$$
\sin \alpha=2 n \sigma_{1} / \pi
$$

also satisfies the equations, if

$$
\kappa^{2}=\frac{9}{16} \omega^{2}+\left(2 \lambda^{0} T_{2} L\right)^{2} \frac{9 n^{2} m^{2}}{4 \pi^{2}} .
$$

Here $\phi_{1}, \phi_{2}$ are again constants, and the parameters $n, m$ are integers. Note that Eq. (27) describes a circle wrapping $n$-times along a meridian of $V_{5,2}$, if all other coordinates were constants. Strictly speaking Eq. (27) makes sense only if $0 \leq \sigma_{1} \leq \pi / 2 n$, since $0 \leq \alpha \leq \pi / 2$. 
We can however continuously extend the geodesic up to $\sigma_{2}=2 \pi$ as we do with $\theta$, while we traverse along the great circle $n$-times.

For conserved quantities, we again find $J_{A}=J_{B}=0$, and a dispersion relation

$$
E^{2}=R^{2}+\left(\frac{3 n m \sqrt{\lambda}}{8 \pi}\right)^{2}
$$

Let us now contemplate on the form of dual operators. First of all, we argue that they must be near-BPS and written as a holomorphic expression for $R>0$ because the dispersion relation approaches the unitarity bound $E \geq R$ as $E, R \rightarrow \infty$. Note that we have effectively identified the two $S^{2}$ 's and the solution is invariant under the exchange of two $S U(2)$ 's. We thus expect there are no monopole operators and we can write schematically the dual operators as $\operatorname{Tr}\left[\Phi^{n_{1}}(A B)^{n_{2}}\right]$, with $n_{1}+2 n_{2}=3 R / 2$. Also recall that among four possible

combinations of $A_{i} B_{j}(i, j=1,2)$, the $S U(2)$ singlet is automatically projected out due to the F-term condition. The fact that we are left with symmetric combinations is in harmony with $\theta_{1}=\theta_{2}$.

\section{Rotating membranes in the subspace $T^{1,1}$}

Let us now consider multi-spin rotating membranes. We will restrict ourselves to the subspace defined by $\alpha=\beta=0$. One can easily check that this is a consistent truncation with all equations. Effectively we are led to study membranes in $\mathbb{R}_{t} \times T^{1,1}$, with the following metric.

$$
\begin{aligned}
\frac{1}{L^{2}} \mathrm{~d} s^{2}= & -\frac{1}{4} \mathrm{~d} t^{2}+\frac{9}{64}\left(\mathrm{~d} \psi+\cos \theta_{1} \mathrm{~d} \phi_{1}+\cos \theta_{2} \mathrm{~d} \phi_{2}\right)^{2} \\
& +\frac{3}{16}\left(\mathrm{~d} \theta_{1}^{2}+\sin ^{2} \theta_{1} \mathrm{~d} \phi_{1}^{2}+\mathrm{d} \theta_{2}^{2}+\sin ^{2} \theta_{2} \mathrm{~d} \phi_{2}^{2}\right) .
\end{aligned}
$$

One can compare this with the well-known Sasaki-Einstein metric [21],

$$
d s_{S E}^{2}=\frac{1}{9}\left(\mathrm{~d} \psi+\cos \theta_{1} \mathrm{~d} \phi_{1}+\cos \theta_{2} \mathrm{~d} \phi_{2}\right)^{2}+\frac{1}{6}\left(\mathrm{~d} \theta_{1}^{2}+\sin ^{2} \theta_{1} \mathrm{~d} \phi_{1}^{2}+\mathrm{d} \theta_{2}^{2}+\sin ^{2} \theta_{2} \mathrm{~d} \phi_{2}^{2}\right) .
$$

The relative sizes of the Reeb vector, against the two-spheres within the Kähler-Einstein base, are obviously different. Using the criteria given in Eq. (2.8) of [21], we see that the five-dimensional space in Eq. (30) is not Einstein. Topologically they are both $S^{2} \times S^{3}$.

Imposing $\alpha=\beta=0$ is equivalent to setting $z_{0}=0$ in Eq. 11]. Then we have $\left\{\sum_{i=1}^{4} z_{i}^{2}=\right.$ $0\}$, i.e. a conifold singularity. On the field theory side, due to the mapping $\Phi \rightarrow z_{0}$, we expect the dual operators would not contain any $\Phi$ 's. 
For explicit solutions we will again adopt the temporal gauge and set $t=\kappa \tau$. Then we may write down the conserved charges as follows.

$$
\begin{aligned}
E & =\frac{\sqrt{\lambda^{\prime}}}{4} \kappa \\
R & =\frac{3 \sqrt{\lambda^{\prime}}}{16} \int \frac{\mathrm{d}^{2} \sigma}{(2 \pi)^{2}}\left(\dot{\psi}+\cos \theta_{1} \dot{\phi}_{1}+\cos \theta_{2} \dot{\phi}_{2}\right), \\
J_{A} & =\frac{3 \sqrt{\lambda^{\prime}}}{128} \int \frac{\mathrm{d}^{2} \sigma}{(2 \pi)^{2}}\left[3\left(\dot{\psi}+\cos \theta_{1} \dot{\phi}_{1}+\cos \theta_{2} \dot{\phi}_{2}\right) \cos \theta_{1}+4 \sin ^{2} \theta_{1} \dot{\phi}_{1}\right], \\
J_{B} & =\frac{3 \sqrt{\lambda^{\prime}}}{128} \int \frac{\mathrm{d}^{2} \sigma}{(2 \pi)^{2}}\left[3\left(\dot{\psi}+\cos \theta_{1} \dot{\phi}_{1}+\cos \theta_{2} \dot{\phi}_{2}\right) \cos \theta_{2}+4 \sin ^{2} \theta_{2} \dot{\phi}_{2}\right] .
\end{aligned}
$$

We may find interesting solutions if

$$
\phi_{1}=0, \quad \theta_{1}=n \sigma_{1},
$$

with $n \in \mathbb{Z}$ and all other coordinates are functions of $\left(\tau, \sigma_{2}\right)$ only. Then remaining equations can be alternatively derived from an auxiliary Polyakov string action, moving within a squashed three-sphere

$$
\frac{1}{L^{2}} \mathrm{~d} s^{2}=-\frac{1}{4} \mathrm{~d} t^{2}+\frac{9}{64}(\mathrm{~d} \psi+\cos \theta \mathrm{d} \phi)^{2}+\frac{3}{16}\left(\mathrm{~d} \theta^{2}+\sin ^{2} \theta \mathrm{d} \phi^{2}\right)
$$

This metric is obtained as a subspace of Eq. (30) if we suppress $\theta_{1}, \phi_{1}$ and rename $\sigma_{2}, \theta_{2}, \phi_{2} \rightarrow$ $\sigma, \theta, \phi$ for brevity. The constraint equations Eqs. (17t18) can be interpreted as Virasoro constraints if the string tension is given as

$$
T_{P}=\frac{1}{2 \lambda^{0}}=\frac{\sqrt{3}}{4} n L T_{2} .
$$

The study of classical rotating strings in the squashed three-sphere like Eq. (37) has appeared several times in the context of semiclassical string/membranes [16, 22, 23]. The equations are further reduced to a mechanical problem if we take the following ansatz.

$$
\psi=\omega \tau, \quad \phi=\nu \tau, \quad \theta=\theta(\sigma) .
$$

The equations are either trivial or can be derived from the constraint condition

$$
\theta^{\prime 2}+V(\theta)=\mathcal{E}
$$

with

$$
\mathcal{E}=\frac{4 \kappa^{2}}{3}, \quad V(\theta)=\nu^{2} \sin ^{2} \theta+\frac{3}{4}(\omega+\nu \cos \theta)^{2}
$$


Let us record here the conserved quantities for the ansatz Eq. 36 39. We obtain

$$
\begin{aligned}
& \frac{E}{\sqrt{\lambda}}=\frac{e \kappa}{4}, \\
& \frac{R}{\sqrt{\lambda}}=\frac{3 e}{16} \int \frac{\mathrm{d} \sigma}{2 \pi}(\dot{\psi}+\cos \theta \dot{\phi}), \\
& \frac{J_{A}}{\sqrt{\lambda}}=0 \\
& \frac{J_{B}}{\sqrt{\lambda}}=\frac{3 e}{128} \int \frac{\mathrm{d} \sigma}{2 \pi}\left[3(\dot{\psi}+\cos \theta \dot{\phi}) \cos \theta+4 \sin ^{2} \theta \dot{\phi}\right],
\end{aligned}
$$

where $e=\frac{\sqrt{3} n}{4}$.

The equation almost trivialises if $\nu=0$. Then $V(\theta)$ is constant, periodicity demands $\theta_{2}=m \sigma_{2}(m \in \mathbb{Z})$, and we have toroidal rotating membranes with single spin. Let us just provide the dispersion relation here.

$$
E^{2}=R^{2}+\left(\frac{3 n m \sqrt{\lambda}}{32}\right)^{2}, \quad J_{A}=J_{B}=0
$$

The dual operators are written in terms of $A_{i}, B_{j}$ only, with monopole operators when needed. One can also expect that there must be the same number of $A_{1}$ 's and $A_{2}$ 's, because $J_{A}=0$. The same argument applies to $B_{i}$ 's.

In the following we consider the case of $\nu \neq 0$. The analysis will be very similar to that of [16, 22]. For cosmetic reasons, we define $a=1, b=3 / 4, c=4 / 3, v=\kappa / \nu, \xi=\omega / \nu$. Then the following results can be applied to

$$
V(\theta)=a \nu^{2} \sin ^{2} \theta+b(\omega+\nu \cos \theta)^{2}
$$

with $\mathcal{E}=c \kappa^{2}$.

1. Toroidal and cylindrical rotaing membranes with $\omega=0$

- Toroidal rotating membranes:

For simplicity, we first consider $\omega=0$ case. The potential has a maximum value at $\theta=\pi / 2$. It $c v^{2}<a$ the motion is vibrational, and the membrane is folded along $\theta$. Due to the periodicity condition $\theta(\sigma)=\theta(\sigma+2 \pi)$, we obtain

$$
\nu=\frac{2 e}{\pi \sqrt{a-b}} \mathbf{K}(y) .
$$


In the following we will use $y=\frac{c v^{2}-b}{a-b}$. The nonvanishing charges are given as

$$
\begin{aligned}
\frac{E}{\sqrt{\lambda}} & =\frac{e}{\pi} \sqrt{\frac{y(a-b)+b}{c(a-b)}} \mathbf{K}(y), \\
\frac{R}{\sqrt{\lambda}} & =\frac{2 e}{\sqrt{a-b}}, \\
\frac{J_{B}}{\sqrt{\lambda}} & =\frac{e}{2 \pi \sqrt{a-b}}(4 \mathbf{K}(y)-\mathbf{E}(y)),
\end{aligned}
$$

where $\mathbf{K}(y), \mathbf{E}(y)$ are the first and second complete elliptic integrals.

- Cylindrical rotating membranes :

For $c v^{2} \geq a$ or equivalently $y \geq 0$, we have rotating membranes cylindrical shape. Integration of the equation of motion gives us the following results.

$$
\begin{aligned}
\nu & =\frac{2}{\pi \sqrt{y(a-b)}} \mathbf{K}(1 / y), \\
\frac{E}{\sqrt{\lambda}} & =\frac{e}{\pi} \sqrt{\frac{y(a-b)+b}{c y(a-b)}} \mathbf{K}(1 / y), \\
\frac{R}{\sqrt{\lambda}} & =0, \\
\frac{J_{B}}{\sqrt{\lambda}} & =\frac{e}{2 \pi \sqrt{y(a-b)}}((3+y) \mathbf{K}(1 / y)-y \mathbf{E}(1 / y)) .
\end{aligned}
$$

In the limit $y \rightarrow 1$ the elliptic functions $\mathbf{K}(y)$ develope a logarithmic divergence, and the nonvanishing charges become large. One can express the dispersion relation as a series, using nome- $q$ expansion of elliptic integrals. The result is summarised in the table $\mathrm{I}$.

\section{Multi-spin rotaing membranes with $\omega \neq 0$}

In this subsection we consider the general cylindrical motion of membrane with the potential (41). From the constraint (17) and the periodicity condition $\theta(\sigma+2 \pi)=\theta(\sigma)$ we get

$$
\nu=\frac{2}{\pi \sqrt{a-b}} \int_{0}^{\theta_{0}} \frac{\mathrm{d} \theta}{\sqrt{(\cos \theta-\alpha)(\cos \theta-\beta)}},
$$

where $\theta_{0}=\cos ^{-1} \alpha$ and $\alpha, \beta(\alpha>\beta)$ are two roots of the quadratic equation obtained from the constraint (17). The energy and conserved chargs are expressed in terms of the elliptic 


\begin{tabular}{c|c}
\hline \hline & Toroidal membrane \\
\hline$E$ & $-\frac{3}{16 \pi} \ln q\left(1+2 q+10 q^{2}+\cdots\right)$ \\
$J_{B}$ & $-\frac{3 \sqrt{3}}{64 \pi}\left\{\ln q\left(1+2 q+8 q^{2}+\cdots\right)+\left(\frac{1}{2}-2 q+6 q^{2}+\cdots\right)\right\}$ \\
Dispersion & \begin{tabular}{c}
$E=\frac{4 \sqrt{3}}{3}\left(J_{B}+\frac{3 \sqrt{3}}{128 \pi}\right)-\frac{3}{8 \pi} \exp \left[-\frac{64 \sqrt{3} \pi}{9}\left(J_{B}+\frac{3 \sqrt{3}}{128 \pi}\right)\right]+\cdots$ \\
\hline$E$
\end{tabular} \\
\hline$J_{B}$ & $-\frac{3 \sqrt{3}}{64 \pi}\left\{\ln q\left(1-2 q+8 q^{2}+\cdots\right)+\left(\frac{1}{2}+2 q+6 q^{2}+\cdots\right)\right\}$ \\
Dispersion & $E=\frac{4 \sqrt{3}}{3}\left(J_{B}+\frac{3 \sqrt{3}}{128 \pi}\right)+\frac{3}{8 \pi} \exp \left[-\frac{64 \sqrt{3} \pi}{9}\left(J_{B}+\frac{3 \sqrt{3}}{128 \pi}\right)\right]+\cdots$ \\
\hline
\end{tabular}

TABLE I. Q-series and dipersion relations for $\omega=0$. (we put $\sqrt{\lambda}=1$ and $n=1$.)

integrals as follows.

$$
\begin{aligned}
\frac{E}{\sqrt{\lambda}}= & \frac{e v}{\pi \sqrt{a-b}} \frac{1}{\sqrt{(1+\alpha)(1-\beta)}} \mathbf{K}(t), \\
\frac{R}{\sqrt{\lambda}}= & \frac{3 e}{4 \pi \sqrt{a-b}} \frac{1}{\sqrt{(1+\alpha)(1-\beta)}}[(\xi-1) \mathbf{K}(t)+2 \mathbf{\Pi}(k, t)], \\
\frac{J_{B}}{\sqrt{\lambda}}= & \frac{3 e}{16 \pi \sqrt{a-b}} \frac{1}{\sqrt{(1+\alpha)(1-\beta)}}\left[\left(2-\frac{3}{2} \xi+\frac{\alpha}{2}\right) \mathbf{K}(t)\right. \\
& \left.+\left(3 \xi-\frac{1}{2}(\alpha+\beta)\right) \mathbf{\Pi}(k, t)-\frac{1}{4}(1+\alpha)(1-\beta) \mathbf{E}(t)\right],
\end{aligned}
$$

where we have defined

$$
\begin{aligned}
& k=-\frac{1-\alpha}{1+\alpha} \\
& t=\frac{(1-\alpha)(1+\beta)}{(1+\alpha)(1-\beta)} .
\end{aligned}
$$

We would like to consider the case where the physical quantities become large. If we send $v,-\xi \rightarrow \infty$, we will get regular series expansions of $E$ and $J_{B}$ in terms of $J_{R}$. More concretely, we assume

$$
c v^{2} \sim b\left(\xi^{2}+2 \varepsilon \xi+\cdots\right)
$$

with $-1<\varepsilon<1$. Then there exist folded spinning strings whose energy can be made arbitrarily large. One easily sees $\alpha \rightarrow \varepsilon, \beta \rightarrow-\infty$ and $k, t \rightarrow \frac{\varepsilon-1}{\varepsilon+1}$ in this limit. We will 
obtain series expansions of the following form,

$$
\begin{aligned}
E & =a_{1} R+a_{2} \frac{\lambda}{R}+a_{3} \frac{\lambda^{2}}{R^{3}}+\cdots, \\
J_{B} & =b_{1} R+b_{2} \frac{\lambda}{R}+b_{3} \frac{\lambda^{2}}{R^{3}}+\cdots
\end{aligned}
$$

We have computed first few coefficients,

$$
\begin{aligned}
& a_{1}=1, \\
& a_{2}=\frac{9}{128 \pi^{2}} \mathbf{K}(-z)(\mathbf{E}(-z)-\mathbf{K}(-z)), \\
& b_{1}=\frac{3}{8}-\frac{3}{4(z+1)} \frac{\mathbf{E}(-z)}{\mathbf{K}(-z)},
\end{aligned}
$$

where

$$
z=\frac{1-\varepsilon}{1+\varepsilon} .
$$

Let us consider the dual operators. Eq. (65) implies that the unitarity bound is asymptotically saturated, so we expect the dual operators are made of $A_{i}, B_{i}$ and not their complex conjugates. Since $J_{A}=0$, it is clear that there are the same number of spin-ups $A_{1}$ and spin-downs $A_{2}$. For the composition of $\left(B_{1}, B_{2}\right)$, we have a complicated implicit relation given by the series.

The appearance of elliptic integrals is in fact not unfamiliar in the spectrum of integrable spin chain. For the duality of $\mathcal{N}=4$ super Yang-Mills theory and IIB strings in $\mathrm{AdS}_{5} \times S^{5}$, one can push the computations on both sides of the duality. The coefficient $a_{2}$ encodes $\mathcal{O}(\lambda)$, i.e. one-loop computation which is conveniently summarised as spin system with nearestneighbor interactions. The spinning string result and the Bethe ansatz result show exact match [24]. Although we do not have a viable field theory result since the $\mathcal{A}_{1}$ quiver theory is strongly coupled, it would be very nice if Eqs. 66 67) can be derived from an integrable $S O(5)$ spin chain.

\section{Giant magnon and single spike solutions}

On the string theory side, giant magnons and spiky string solutions are rotating open strings with infinite energy and angular momenta. For the giant magnon solutions on $S^{2}$ the end points are on the equator and carry infinite momenta.

Benvenuti and Tonni studied giant magnons and single spikes in squashed three-sphere [23. Since we have reduced the rotating membrane problem into a Polyakov string moving 
in the squashed three-sphere, the results of [23] are directly applicable here. Instead of repeating all the derivations, we will very briefly explain what kind of solutions we are after, and present the result.

For giant magnons and single spikes, one introduces the following ansatz for string motion in Eq. (37).

$$
t=\kappa \tau, \quad \theta=\theta(x), \quad \psi=\omega \tau+\Psi(x), \quad \phi=\nu \tau+\Phi(x),
$$

where $x=\beta \sigma-\gamma \tau$ and $\beta, \gamma$ are constants.

The equation for $t$ is still trivial. One then looks at the equations for $\psi, \phi$ and find they are nontrivial, unlike the spinning strings we have considered so far. Fortunately they can be integrated once, and one can express $\Psi^{\prime}, \Phi^{\prime}$ as functions of $\theta$. If we plug those relations into the equation of motion for $\theta$, we obtain a mechanical system with $\theta^{\prime 2}+V(\theta)=0$. Giant magnons and spiky strings are characterised by the condition $V(\theta) \propto-(\theta-\pi)^{2}$ which implies it takes infinitely long to arrive at the turning point $\theta=\pi$. In terms of strings, this means the open string end points lie at $\theta=\pi$. The giant magnons are U-shaped and characterized by $\Delta \phi$ which is the difference of $\phi$ coordinate at the two end points. With spiky strings $\Delta \phi$ is divergent as well as energy.

The analysis of [23] is for general squashed three-sphere with $S U(2) \times U(1)$ symmetry. We can translate their result for our setting with simple re-scalings. We will just record the dispersion relations. For simplicity we set $\sqrt{\lambda}=1$.

For giant magnon type solutions, $E, R, J_{B}$ are all infinite but the linear combinations $E-R, E-8 J_{B} / 3$ are finite. The dispersion relations is

$$
E-\frac{8}{3} J_{B}=\frac{n}{2} \frac{\cos (4(E-R) / 3 n)-\cos \Delta \varphi}{\sin (4(E-R) / 3 n)} .
$$

For a single spike, $\Delta \phi, E$ are divergent but $R, J_{B}$ are finite. The dispersion relation is

$$
J_{B}=\frac{3 n}{16} \frac{\cos (8 E / 3 n-\Delta \varphi)-\cos (4 R / 3 n)}{\sin (4 R / 3 n)} .
$$

\section{DISCUSSION}

We have studied rotating membranes in nono-toric Sasaki-Einstein manifold $V_{5,2}$. The metric is still homogeneous, but more complicated than $Q^{1,1,1}$ or $M^{1,1,1}$ which are toric. At first sight, probably only the $S U(2) \times S U(2) \times U(1)_{R}$ part of the isometry is manifest. 
The main task in this article is to construct explicit solutions and provide their field theory interpretations as long operators. We have five alphabets, i.e. chiral multiplets to be used in the construction of chiral primaries. They are $\Phi, A_{1}, A_{2}, B_{1}, B_{2}$ and the last four fields parametrize the manifest $S U(2) \times S U(2)$ symmetry. We managed to find a couple of wrapped membrane solutions which are dual to operators containing $\Phi$ 's. More sophisticated

solutions are constructed in the $T^{1,1}$ subsector. We expect the dual operators are then made of $A_{i}, B_{i}$ and do not contain $\Phi$ 's.

Eventually we would like to have some field theory side computations and compare them against our results in this paper. Since the dual field theory is at a nontrivial fixed point of the renormalization group, conventional field theory techniques are not applicable. According to the standard AdS/CFT dictionary, the classical membrane results correspond to all-order results in the planar limit. It would be nice if we could derive from the membrane solutions or the Hamiltonian operator in the radial quantization prescription of conformal field theory.

\section{ACKNOWLEDGMENTS}

N. Kim is very grateful to D. Martelli for helpful discussions and comments. J. Kim has been partly supported by Institute for the Early Universe (IEU) at Ewha university during the later stages of this work. This research is supported by the National Research Foundation of Korea (NRF) funded by the Korea government (MEST) with the grant No. 2009-0085995 and also partly through the Center for Quantum Spactime (CQUeST) of Sogang University with grant No. R11-2005-021.

\section{Appendix A: Useful formulas for elliptic integrals}

In this appendix we present some definitions and relations which is needed to obtain our results in this note. The complete elliptic integrals of the first and the second is defined as 
below:

$$
\begin{aligned}
\mathbf{K}(m) & =\int_{0}^{\pi / 2} \frac{d \phi}{\sqrt{1-m \sin ^{2} \phi}}, \\
\mathbf{E}(m) & =\int_{0}^{\pi / 2} \sqrt{1-m \sin ^{2} \phi} d \phi \\
\mathbf{\Pi}(k, m) & =\int_{0}^{\pi / 2} \frac{d \phi}{\left(1-k \sin ^{2} \phi\right) \sqrt{1-m \sin ^{2} \phi}} .
\end{aligned}
$$

We often use the following relation

$$
I(n)=\int_{\alpha}^{1} \frac{s^{n} d s}{\sqrt{(s-\alpha)(s-\beta)\left(1-s^{2}\right)}} \quad(\beta<\alpha<1),
$$

which is used to derive the following relations

$$
\begin{aligned}
& I(0)=\frac{2}{\sqrt{(1+\alpha)(1-\beta)}} \mathbf{K}(t), \\
& I(1)=\frac{2}{\sqrt{(1+\alpha)(1-\beta)}}[2 \boldsymbol{\Pi}(k, t)-\mathbf{K}(t)], \\
& I(2)=\frac{1}{\sqrt{(1+\alpha)(1-\beta)}}[2(\alpha+\beta) \mathbf{\Pi}(k, t)-2 \alpha \mathbf{K}(t)+(1+\alpha)(1-\beta) \mathbf{E}(t)],
\end{aligned}
$$

where

$$
k=-\frac{1-\alpha}{1+\alpha}, \quad t=\frac{(1-\alpha)(1+\beta)}{(1+\alpha)(1-\beta)} .
$$

In order to study the elliptic integral near the logarithmic singularities, it is convenient to use the q-series and defined as

$$
\begin{aligned}
q & \equiv \exp [-\pi \mathbf{K}(1-m) / \mathbf{K}(m)] \\
& =\frac{m}{16}+8\left(\frac{m}{16}\right)^{2}+84\left(\frac{m}{16}\right)^{3}+\cdots
\end{aligned}
$$

Inverting the above relation, one obtains

$$
m=16\left(q-8 q^{2}+44 q^{3}-192 q^{4}+\cdots\right)
$$

Then one has the following alternative expansions of elliptic integrals.

$$
\begin{aligned}
& \mathbf{K}(m)=\frac{\pi}{2}\left(1+4 q+4 q^{2}+4 q^{4}+\cdots\right), \\
& \mathbf{E}(m)=\frac{\pi}{2}\left(1-4 q+20 q^{2}-64 q^{3}+\cdots\right) .
\end{aligned}
$$


And more importantly,

$$
\begin{aligned}
& \mathbf{K}(1-m)=-\frac{\ln q}{2}\left(1+4 q+4 q^{2}+4 q^{4}+\cdots\right), \\
& \mathbf{E}(1-m)=\left(1-4 q+12 q^{2}-32 q^{3}+\cdots\right)-4 q \ln q\left(1-2 q+8 q^{2}+\cdots\right) .
\end{aligned}
$$

To get the expansion of $\mathbf{E}(1-m)$ from the above, it is convenient to use the Legendre's relation,

$$
\mathbf{E}(m) \mathbf{K}(1-m)+\mathbf{E}(1-m) \mathbf{K}(m)-\mathbf{K}(m) \mathbf{K}(1-m)=\frac{\pi}{2}
$$

[1] O. Aharony, O. Bergman, D. L. Jafferis, and J. Maldacena, JHEP, 10, 091 (2008), arXiv:0806.1218 [hep-th].

[2] Y. Imamura and K. Kimura, Prog. Theor. Phys., 120, 509 (2008), arXiv:0806.3727 [hep-th],

[3] D. Martelli and J. Sparks, Phys. Rev., D78, 126005 (2008), arXiv:0808.0912 [hep-th],

[4] A. Hanany and A. Zaffaroni, JHEP, 10, 111 (2008), arXiv:0808.1244 [hep-th].

[5] Y. Imamura and K. Kimura, JHEP, 10, 114 (2008), arXiv:0808.4155 [hep-th].

[6] A. Hanany, D. Vegh, and A. Zaffaroni, JHEP, 03, 012 (2009), arXiv:0809.1440 [hep-th],

[7] S. Franco, A. Hanany, J. Park, and D. Rodriguez-Gomez, JHEP, 12, 110 (2008), arXiv:0809.3237 [hep-th].

[8] S. Franco, I. R. Klebanov, and D. Rodriguez-Gomez, JHEP, 08, 033 (2009), arXiv:0903.3231 [hep-th].

[9] M. Aganagic, (2009), arXiv:0905.3415 [hep-th],

[10] D. Martelli and J. Sparks, JHEP, 12, 017 (2009), arXiv:0909.2036 [hep-th].

[11] S. Elitzur, A. Giveon, and D. Kutasov, Phys. Lett., B400, 269 (1997), arXiv:hep-th/9702014.

[12] S. Franco et al., JHEP, 01, 128 (2006), arXiv:hep-th/0505211.

[13] J. Davey, A. Hanany, N. Mekareeya, and G. Torri, (2009), arXiv:0910.4962 [hep-th],

[14] S. S. Gubser, I. R. Klebanov, and A. M. Polyakov, Nucl. Phys., B636, 99 (2002), arXiv:hepth/0204051.

[15] P. Bozhilov, JHEP, 08, 087 (2005), arXiv:hep-th/0507149, 03, 001 (2006), arXiv:hepth/0511253 (2006), arXiv:hep-th/0612175 Phys. Rev., D76, 106003 (2007), arXiv:0706.1443 [hep-th]; JHEP, 08, 073 (2007), arXiv:0704.3082 [hep-th]; Fortsch. Phys., 56, 373 (2008), arXiv:0711.1524 [hep-th]; P. Bozhilov and R. C. Rashkov, Nucl. Phys., B794, 
429 (2008), arXiv:0708.0325 [hep-th]; W.-Y. Wen, B791, 164 (2008), arXiv:0705.2634 [hep-

th]; C. Ahn and P. Bozhilov, JHEP, 08, 054 (2008), arXiv:0807.0566 [hep-th].

[16] J. Kim, N. Kim, and J. H. Lee, (2010), arXiv:1001.2902 [hep-th],

[17] A. Ceresole, G. Dall'Agata, R. D'Auria, and S. Ferrara, JHEP, 03, 011 (2000), arXiv:hepth/9912107.

[18] D. M. Hofman and J. M. Maldacena, J. Phys., A39, 13095 (2006), arXiv:hep-th/0604135.

[19] M. Kruczenski, JHEP, 08, 014 (2005), arXiv:hep-th/0410226.

[20] A. Bergman and C. P. Herzog, JHEP, 01, 030 (2002), arXiv:hep-th/0108020.

[21] P. Candelas and X. C. de la Ossa, Nucl. Phys., B342, 246 (1990).

[22] N. Kim, Phys. Rev., D69, 126002 (2004), arXiv:hep-th/0312113.

[23] S. Benvenuti and E. Tonni, JHEP, 02, 041 (2009), arXiv:0811.0145 [hep-th].

[24] N. Beisert, S. Frolov, M. Staudacher, and A. A. Tseytlin, JHEP, 10, 037 (2003), arXiv:hepth/0308117. 